\section{Sistemas de Información en Salud: de sistemas cerrados a la ciudadanía social. Un desafío en la reducción de desigualdades en la gestión local}

\author{
Health Information Systems: from closed systems \\ to social citizenship. A challenge for the reduction \\ of inequalities in local management
}

\author{
Marcio Alazraqui 1 \\ Eduardo Mota 2 \\ Hugo Spinelli 1
}

\section{Maestría en Epidemiología, Gestión y Políticas de Salud, Universidad Nacional de Lanús, Lanús, Argentina. 2 Instituto de Saúde Coletiva, Universidade Federal da Bahia, Salvador, Brasil. \\ Correspondencia M. Alazraqui Maestría en Epidemiología, Gestión y Políticas de Salud, Universidad Nacional de Lanús. \\ Maza 1966, Ciudad Autónoma de Buenos Aires C1240ADP, Argentina. alazra@mail.retina.ar}

\begin{abstract}
The traditional concept of health information systems (HIS) poses numerous problems when attempting to support local management orientated to the reduction of health inequalities. How does one design a local HIS, and what would its characteristics be? We view HIS as open and complex systems of which we ourselves are a part. The hypothesis is that a HIS that provides support to local management must be conceived as a set of processes including data, information, knowledge, communication, and action (DIKCA). Data constitute a complex structure with five components. Information is a set of processed data; meanwhile knowledge output involves a subject's understanding and grasp of the phenomenon. Communication links the previous concepts to action. Strategic and communicative actions should be priorities in local management. This proposal aims at management support by the HIS to eliminate health inequalities and build an inclusive society.
\end{abstract}

Health Inequalities; Information Systems; Management

\section{Introducción}

El Sistema de Información en Salud (SIS), según la Oficina Regional de la Organización Mundial de la Salud (OMS), "é um mecanismo de coleta, processamento, análise e transmissão da informação necessária para se organizar e operar os serviços de saúde e, também, para a investigação e o planejamento com vistas ao controle de doenças" 1 (p. 26). Esta definición de SIS merece algunas reflexiones. El gran desarrollo de la informática en los últimos años ha permitido trabajar con volúmenes muy grandes de datos e información, así como transmitirlos sin dificultades. En este sentido, se nota un avance muy importante en la resolución de problemas de orden normativo. Sin embargo, los problemas de los SIS persisten y se centran en el poco avance en su implementación y utilización como soporte de la gestión.

Identificamos múltiples problemas en los SIS que condicionan y limitan fuertemente su aplicación en la gestión y que están relacionados con las siguientes concepciones: (a) totalidad simple, o unidad concebida como simplicidad; (b) estabilidad, como cualidad de estático; y (c) obediencia estricta a normas con respuestas previsibles. Estos aspectos llevan a concebir a los SIS como sistemas simples y cerrados, tal como se puede apreciar en la definición de la OMS. Esta concepción general explica a nuestro entender una serie de problemas graves en su utilización por la gestión que explicitaremos más adelante. 
Otra forma de concebir a los SIS es considerarlos como sistemas abiertos y complejos. Esta concepción tiene antecedentes en la teoría general de los sistemas, formulada inicialmente por Bertalanffy 2 . Esta teoría postula cuatro características básicas de un sistema: (a) totalidad, de tal forma que los elementos en conjunto son diferentes a la suma de las partes; (b) límites, en el sentido de sistema abierto que mantiene una relación con el contexto que lo rodea; (c) jerarquía, en que cada sistema está formado por subsistemas y forma parte a su vez de suprasistemas; y (d) equifinalidad, ya que un sistema puede alcanzar un punto determinado de desarrollo a partir de diferentes condiciones iniciales 2,3.

El desarrollo de la cibernética ha enriquecido la teoría general de los sistemas. El término cibernética viene del griego kybernçtiké y significa "el arte de gobernar". Originalmente definida como la ciencia del control y la comunicación en sistemas complejos, se orienta actualmente al estudio de las relaciones de organización que deben tener los componentes de un sistema para existir como una entidad autónoma 4. En relación al investigador podemos dividir la cibernética de primer o segundo orden, según Behncke 4 (p. xx): "Al estudio de los sistemas supuestamente 'independientes' de nuestra actividad cognoscitiva (de observación), se le llamó cibernética de primer orden, o cibernética de los sistemas observados, puesto que el observador se supone marginado de tal sistema; al estudio de los sistemas en los cua- les nuestra propia actividad descriptiva es parte constitutiva de los mismos se le llamó cibernética de segundo orden, o cibernética de los sistemas observadores".

La cibernética de segundo orden a su vez fue un gran salto en la concepción de esta disciplina 5. Los SIS por sus características deben concebirse dentro de esta concepción.

La concepción de los SIS como sistemas abiertos, complejos y en los que somos parte constitutiva, nos permite comprender problemas actuales de los sistemas de información y orientar acciones para enfrentarlos. Esta concepción es más acorde también con una concepción de salud en el sentido amplio, y no solamente limitada a lo médico.

Concebimos al SIS como un sistema jerárquico formado por otros subsistemas. Sus principales subsistemas son los siguientes: sistema de información epidemiológica, sistema de información económica financiera y administrativa, sistema de información sociodemográfica y social, y sistema de información de carácter clínico 6 (Figura 1).

Para abordar la lógica procesual del SIS deberemos discutir los procesos de producción y aplicación de información en salud. Es decir, ¿qué caracteriza a estas etapas y cuál es la relación entre ellas?

Un sistema de información que pueda dar soporte a la gestión local debe articular los siguientes elementos: dato, información, conocimiento,

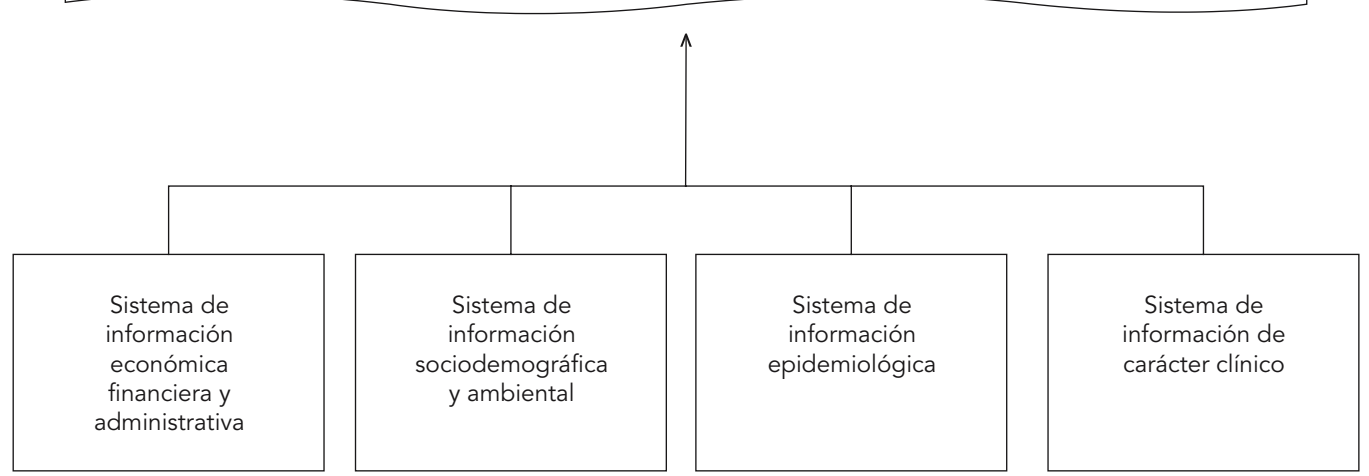


comunicación y acción. Todos ellos orientados al concepto de ciudadanía social. Identificamos en la secuencia anterior componentes tradicionales como dato, información y conocimiento; y nuevos conceptos como los de comunicación y acción.

Estos componentes se entienden como un conjunto, constituyendo una lógica de procesos, y no como compartimentos estancos. Conforman básicamente dos subgrupos, uno relacionado con la producción y el otro con la aplicación de la información, aunque estos límites a veces no son tan claros. El componente tradicional de carácter más normativo está relacionado con la producción: dato e información. El componente de aplicación de carácter estratégico comunicacional está formado por: conocimiento, comunicación y acción.

La problemática anterior nos lleva a la pregunta, ¿̇ómo diseñar un sistema de información en el nivel local y cuáles son sus características? Asumimos que la forma tradicional de concepción de los SIS no es pertinente para dar soporte a la gestión, ni apoyar acciones para la reducción de desigualdades. La hipótesis es que la única forma de construir un sistema de información en salud que dé soporte a la gestión local es concebirlo como un sistema comple- jo que debe ser abordado a partir del conjunto de elementos formado por datos, información, conocimiento, comunicación y acción (DICCA) (Figura 2).

A continuación identificaremos problemas en los SIS aplicados a la gestión local en salud; y luego discutiremos aspectos relevantes de los elementos centrales de la propuesta a fin de desarrollar una línea de trabajo en SIS que apoye nuestro trabajo en desigualdades, donde hemos identificado a los SIS como un problema. Muchos de estos problemas no son exclusivos de la gestión local y se encuentran en la gestión en general también; pero como nuestra intención es problematizar la acción local nos referimos a ella más específicamente.

\section{Problemas de los Sistemas de Información en Salud (SIS)}

Debido al gran desarrollo de sistemas informatizados podemos decir que la capacidad actual de almacenar y enviar datos e información en formato electrónico es muy grande y no constituye un problema en sí. El problema son las respuestas a preguntas como: ¿icuáles datos?, ¿̇por qué?, ¿de qué forma?, ¿para qué?, ¿en qué periodicidad?,

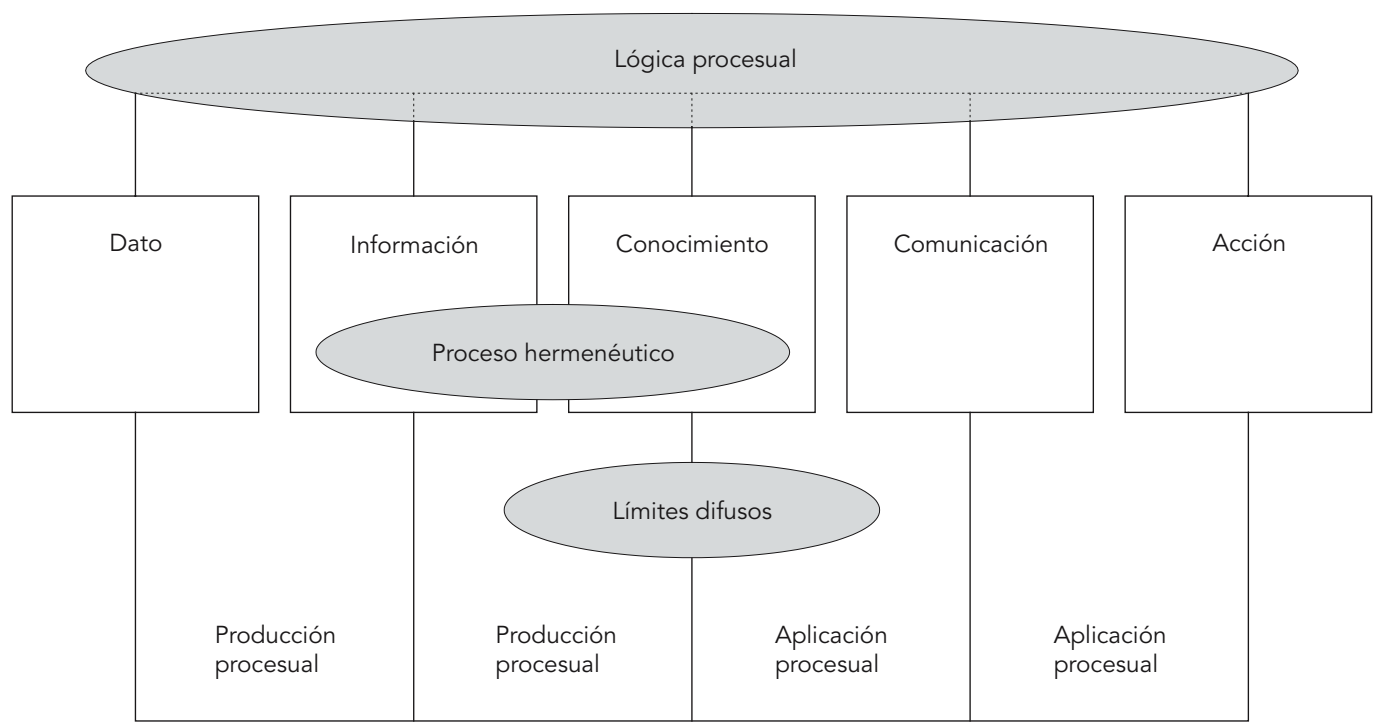


¿cómo se realiza el control de su calidad?, ¿cómo se evalúan?, ¿cómo se protege la confidencialidad de las personas involucradas?, entre otras.

Cada día es mayor el volumen de datos e información en salud existentes, así como cada día son mayores las dificultades para lidiar con ellos de forma que permitan orientar acciones operativas. Dificultades que se expresan en términos de selección, jerarquización, interpretación y apropiación por el sujeto. Es decir, en cuestiones que no se resuelven con el desarrollo informático mencionado anteriormente. Castiel \& Vasconcellos-Silva 7 denominan "precariedades del exceso" a este aspecto paradójico, representado por una abundancia de información de difícil utilización, tanto por su volumen como por su calidad.

Existen varios problemas en la producción de información en salud en el nivel local que repercuten en la calidad de la información y dificultan su aplicación en la gestión. Estos problemas dependen, en diferentes medidas, del nivel local y/o de niveles superiores.

En América Latina encontramos una serie de problemas en los datos y la información sobre condiciones de vida y eventos del Proceso Salud Enfermedad Cuidado (PSEC) que dificultan su utilización. Estos problemas son: registros asistemáticos, ausencia de soporte magnético (en formato papel), bases de datos sin formalización institucional (falta de reconocimiento formal de la institución), superposiciones e incoherencias entre bases de datos semejantes, entre otros problemas. En definitiva esta información presentada así es de muy baja utilidad en la toma de decisiones y como consecuencia insuficiente para dar soporte a la gestión.

En relación a la capacitación encontramos habitualmente que los datos recolectados no son utilizados para producir información que dé soporte a la intervención, y pasan a tener una "función administrativa predominante" en la unidad de información local. La falta de utilización, o subutilización, de los datos en el nivel local origina un círculo vicioso donde las unidades de información locales producen datos de mala calidad que excepcionalmente utilizan. Por otro lado, el envío de datos al nivel central habitualmente no es seguido de una devolución de los mismos, enfocada a la evaluación de su calidad y en su aplicación en el nivel de origen. La mayoría de los SIS continuos y obligatorios tienen como nivel operacional el nivel local. En estos casos el nivel nacional es un receptor de datos locales que procesa y publica, en forma de datos agregados.

En las unidades de información centrales se puede observar claramente la calidad heterogénea de los datos recolectados. Esta heterogeneidad se debe a diversas realidades locales con di- ferentes estructuras socioeconómicas, capacidades organizativas, capacidades administrativas y de prestación de servicios, por ejemplo. Desde ya el problema no se limita a las organizaciones responsables de la producción de información "per se", sino a que estas son un reflejo de los actores sociales involucrados y sus intereses 1 .

Las necesidades de información en salud cambian a lo largo del tiempo en relación a cambios en la situación social del espacio considerado 8. Estas nuevas necesidades de información obligan a modificar, agregar y eliminar datos en nuestras fuentes de información.

A fin de abordar y caracterizar los problemas encontrados en el nivel local y su relación con la propuesta de abordaje de los SIS, a continuación presentaremos aspectos relevantes para cada uno de ellos.

\section{Sistema de información en salud en el nivel local: propuesta DICCA}

La producción de información en salud está tradicionalmente pensada según el esquema "dato, información y conocimiento” (DIC). Este esquema es insuficiente para orientar la aplicación de información en salud en el nivel local con el objetivo de eliminar/reducir desigualdades en salud. Se propone como orientador de la producción y aplicación de información en salud en el nivel local el esquema "dato, información, conocimiento, comunicación y acción” (DICCA). Este esquema nos permitirá explicitar una propuesta a fin de abordar la hipótesis precedente.

¿Qué queremos decir con DICCA? Si queremos construir un sistema de información para la gestión necesitamos incluir todos los elementos mencionados en DICCA. Entendemos este conjunto de elementos en una lógica procesual, donde mantienen entre sí una interrelación múltiple y de retroalimentación, es decir, no tienen una relación lineal. Sostenemos que si logramos desarrollar un proceso de trabajo que incluya los cinco elementos estaremos en presencia de un sistema de información para la gestión.

Aunque los términos dato, información y conocimiento se utilicen habitualmente sin definiciones muy precisas, y con ciertas superposiciones entre ellos, existen esfuerzos en delimitar sus diferencias. Discutiremos las definiciones de los términos utilizados y luego explicaremos por qué los entendemos como un proceso.

\section{$\underline{\text { Dato }}$}

Moraes 1 (p. 19) define dato de la siguiente forma, "é uma descrição limitada do real, desvin- 
culada de um referencial explicativo e difícil de ser utilizada como informação por ser inintelegível". Consideramos al dato en esta investigación, como una estructura compleja formada por tres elementos básicos: las unidades de análisis, las variables y los valores de las variables. Galtung 9 (p. 1) define de la siguiente manera cada uno de los componentes del dato: "En primer lugar, y es lo más importante, tenemos los elementos de análisis o unidades de análisis, tales como seres humanos (interpelados) en una encuesta o en un experimento de laboratorio, o naciones en un estudio comparativo de las naciones, o unidades más complejas. (...) En segundo lugar, existen las dimensiones o variables, que uno desea conocer en cuanto a las unidades, tales como el consumo de energía per cápita, en los estudios acerca de las naciones subdesarrolladas, o las respuestas a ciertas preguntas estratégicas en un estudio acerca de la elección. (...) En tercer lugar, tenemos los valores que alcanzan las unidades en las variables estudiadas o, utilizando las otras expresiones, las respuestas o los resultados que se obtienen cuando las unidades se exponen a los estímulos o a las condiciones según las cuales el sociólogo desea estudiarlas".

El principal problema observado en la unidad de análisis es conceptual y se refiere a considerar al individuo de forma aislada. En cuanto a las variables y a los valores de las variables encontramos cuestiones relacionadas con la completitud y con el correcto llenado, entre otras. Varios autores describen dificultades semejantes a las señaladas en el nivel local 10,11,12,13.

Hemos definido inicialmente el dato como una estructura compleja tripartita ${ }^{9}$. Samaja 14 propone el dato como una estructura compleja integrada por cinco ingredientes lógicos, que nos parece propicia para la comprensión del tema. Según Juan Samara 14 (p. 160), “... un dato es una construcción compleja que, por consecuencia, posee una estructura interna. Esta estructura es su contenido formal invariable (es decir, está presente en todo dato)". Samaja agrega a los tres componentes descritos por Galtung los dos siguientes: indicador y unidad de contexto ${ }^{15}$ La definición de indicador es la siguiente 15 (p. 176): "Un 'indicador' es, en general, algún procedimiento que se lleva a cabo en alguna dimensión (o interpretación particular) de la variable, a fin de determinar qué valor de la variable le corresponde a cada unidad de análisis. Dado que cualquiera que sea la unidad que se tome en cuenta, ella consiste en una totalidad, entonces, todo indicador implicará atender a las partes, las que, en la jerga metodológica, se denominan sub unidades".

Además, Samaja propone concebir la "matriz de datos" como "sistemas de matrices de datos".
De forma simplificada el investigador se posiciona sobre una matriz de datos que considera su nivel de anclaje. Este término hace referencia a que la investigación está "anclada" en ese nivel entre otros posibles. Por encima de este nivel tendría una nueva y diferente matriz de datos constituida por los contextos y denominada "matriz supraunitaria". A su vez, por debajo de su nivel de anclaje tendría otra matriz de datos constituida por los componentes (o partes) de las unidades de análisis del nivel de anclaje, que es denominada "subunitaria" 14. Ambas matrices, superior e inferior, se encuentran conectadas en el nivel de anclaje. De esta forma, este conjunto de matrices, o "sistemas de matrices de datos" conforman un esquema complejo, más acorde con el abordaje de un problema de salud porque integra diferentes niveles de hechos 14,16. El nexo entre estos diferentes niveles está dado por los dos ingredientes del dato mencionados: la unidad de contexto (supra unidad) y el indicador (sub unidad) 15.

¿Por qué son tan importantes los datos? Porque conllevan por lo menos dos cuestiones relevantes: ¿qué datos debemos utilizar en función a los objetivos deseados?, y zquiénes son los que definen qué datos se recolectan? En la producción de información en salud es muy relevante la discusión sobre la elección de qué datos compondrán el instrumento de captura de datos. Esto lleva a la discusión de los objetivos de la producción de información en salud; y estos a su vez a la discusión de los "constructos teóricos" (concepciones teóricas) de los datos y de cada uno de sus componentes, por ejemplo, en la definición conceptual y operativa de las variables. El momento de la elección y definición de qué datos necesitamos es el momento donde nuestros conceptos teóricos se transforman en conceptos operativos a ser aplicados en terreno (transformación del dato conceptual al operativo).

Se observa una cierta tendencia a centrar la discusión en la obtención de nuevos datos con la ilusión de que el "dato ideal", o la profusión de datos, "solucione" los problemas de los SIS.

\section{Información}

Encontramos una ambigüedad en el uso del término información que origina una cierta polisemia en torno al mismo. Esta polisemia está relacionada con un fuerte desarrollo del término en su aplicación a campos muy variados.

Es frecuente el uso indistinto entre los conceptos de información y conocimiento, sin embargo, no son sinónimos. Asumimos que información está relacionada a la noción de "noticia" o "testimonio"; mientras que conocimiento lo está a la noción de "entendimiento" o "compren- 
sión” 17. Para nosotros el conocimiento es una experiencia individual y/o colectiva que no se da necesariamente por mayor que sea la información de que se disponga. Por ejemplo, se puede tener información de la fórmula $\mathrm{E}=\mathrm{mc}^{2}$ correspondiente a la teoría de la relatividad, aunque no se conozca la teoría mencionada, en el sentido de entender o comprender la misma.

Como mencionamos no existe un consenso en la definición de información. Moraes ${ }^{1}$ (p. 19 20) define información de la siguiente forma: " $E$ uma descrição mais completa do real associada a um referencial explicativo sistemático. Pode-se dizer que é a representação de fatos da realidade com base em determinada visão de mundo, mediante regras de simbologia. É portanto, a 'ponte' entre os fatos da realidade ou as idéias de algumas pessoas e as idéias ou conhecimientos de outras".

$\mathrm{Al}$ término información le damos el sentido de un conjunto de datos procesados. Esta definición, arbitraria por cierto, pretende diferenciar información de conocimiento con el objetivo de entender los diferentes componentes procesuales existentes en un SIS y proponer acciones.

\section{Conocimiento}

En la producción de conocimiento existe en general un proceso hermenéutico. Por eso a partir de la misma información se pueden producir distintos conocimientos. Estas diferencias no son mejores ni peores, sino que se relacionan al carácter interpretativo del conocimiento que es realizado por un determinado actor en relación a su referencial teórico y/o ideológico.

En el conocimiento es primordial el concepto de comprensión del fenómeno en el mundo, y de apropiación por parte del sujeto. Es decir, que reconocemos en el conocimiento dentro de la secuencia DICCA un doble carácter; por un lado uno más ligado a la producción (técnica); por el otro el más ligado a la acción y a la constitución del sujeto (pensamiento). En este proceso de producción y aplicación del conocimiento el poder es central como categoría de análisis, tal como lo desarrolla Moraes 18.

Finalmente, si nos quedamos en la información, nos quedamos en la lógica instrumental, en la técnica. La técnica al desplazar al pensamiento niega al sujeto. El conocimiento en tanto proceso hermenéutico rompe con la razón instrumental, y nos permite pasar del ¿cómo se hace?, al ¿por qué se hace?

\section{Comunicación}

¿Por qué la comunicación está incluida en la propuesta? Si nos limitamos a los SIS tradicionales, nos limitamos a DIC y quedamos encerrados en una lógica normativa. Existen varias experiencias en las organizaciones que muestran el fracaso de adherirse a esta lógica exclusivamente, y las críticas al método CENDES/OPS (Centro de Estudios del Desarrollo Económico Social/Organización Panamericana de la Salud) realizadas por Testa 19 lo muestran claramente. Se entiende lo normativo como un momento dentro de la planificación, en relación con otros momentos también significativos 20. Por lo cual no es viable gestionar la producción y aplicación de información en salud solamente con un pensamiento normativo.

La concepción tradicional de la comunicación puede ser representada con la metáfora del "tubo". Algo que se genera en un punto (emisor), es conducido por un conducto (tubo), y se entrega al otro extremo (receptor). Según Maturana 21 (p. 130) esta metáfora es falsa porque supone: “... una unidad no determinada estructuralmente, donde las interacciones son instructivas, como si lo que pasa a un sistema en una interacción quedase determinado por el agente perturbante y no por su dinámica estructural. Sin embargo, es evidente, aun en la vida cotidiana misma, que la situación de comunicación no se da así: cada persona dice lo que dice u oye lo que oye según su propia determinación estructural. Desde la perspectiva de un observador siempre hay ambigüedad en una interacción comunicativa. El fenómeno de comunicación no depende de lo que se entrega, sino de lo que pasa con el que recibe. Y esto es un asunto muy distinto a 'transmitir información'”.

$\mathrm{Al}$ incorporar el concepto de "acto de habla" el hecho comunicacional se hace complejo y los conceptos se pueden utilizar de forma más dinámica ${ }^{22}$. El acto de habla es el módulo básico de las significaciones en un discurso. Los actos ilocutivos son los actos de habla en el sentido restricto de la palabra. Conversamos y escribimos combinando actos de habla ilocutivos. Estos se refieren a aserciones, expresiones, declaraciones, directivas y compromisos. Mientras los actos perlocutivos son los efectos que nuestros actos ilocutivos producen en nuestros interlocutores, como por ejemplo convencer, persuadir o divertir 22,23.

Nuestra comprensión de la comunicación nos permite articular el "mundo de los objetos" al "mundo de los sujetos", es decir, pasar de una lógica normativa a una lógica donde intervienen sujetos con sus intencionalidades y sus conocimientos derivados del análisis e interpretación de la información. Por ello, en este punto es central como categoría de análisis la acción comunicativa.

Concebimos a las organizaciones como una red de conversaciones, es decir, que se confor- 
man a través del lenguaje 24 y este tiene como característica fundamental la intencionalidad 22.

Es en este sentido que los trabajadores dentro de una organización pueden idealmente realizar las siguientes cuestiones: ¿cuáles son nuestros objetivos como organización?, ¿qué vamos a hacer y cómo?, y ża partir de qué procesos de trabajo? A través de la comunicación se debiera construir viabilidad para las acciones y/o consenso con otros actores, entre ellos las organizaciones sociales.

La comunicación es un elemento generalmente poco jerarquizado en las organizaciones. En general existe una preocupación muy central por la cuestión del poder. No restamos importancia a la misma, aunque no la concebimos como eje central único. En las organizaciones intervienen cuestiones relacionadas con el poder y también con la comunicación 25. Hemos abusado en el uso de lo estratégico en relación a acciones comunicativas y preocupados con la cuestión del poder hemos relegado el uso de la comunicación 26. Sin ser explicitado de tal manera esto se vislumbra en las últimas obras de Matus 27 y Testa 28 , donde la centralidad de la categoría poder empieza a dar lugar a las categorías comunicación y lenguaje respectivamente. A final la comunicación está involucrada tanto en el conjunto de acciones estratégicas como en acciones comunicativas.

Estos motivos nos llevan a pensar que la concepción de organización como una red de conversaciones está ligada a la calidad de las mismas. Flores 23 se refiere a la calidad de una organización según la calidad de las conversaciones que se realizan en ella. De esta forma las divide en conversaciones de alta o de baja calidad. Las conversaciones de alta calidad generan compromisos y las acciones correspondientes a los mismos. Las cuales se relacionan indefectiblemente al proceso de trabajo.

\section{Acción}

El proceso DICC (dato, información, conocimiento y comunicación) desarrollado hasta ahora debiera dar soporte a una acción "ligada al acto" de forma tal que disminuya la incertidumbre vinculada a su ejecución, para ello lo que buscamos es lograr una continuidad entre el SIS y la acción a través del DICCA incorporado en el proceso de trabajo y/o de gestión.

¿Cuáles son las acciones a qué hacemos referencia? Acciones estratégicas, o sea orientadas al éxito, y acciones comunicativas, o sea orientadas al entendimiento. Estamos considerando aquí el trabajo cotidiano en dos dimensiones: acción productiva (racionalidad estratégica dirigida a fines) y acción comunicativa (interacción social dirigida al entendimiento) 29 .

Debido a que nuestro objetivo tiene como centralidad la disminución de desigualdades en la gestión local consideramos muy relevante problematizar las acciones desarrolladas de forma cotidiana y que constituyen el proceso de trabajo de los trabajadores de salud. Este proceso de trabajo a su vez está relacionado muy fuertemente con los modelos de cuidado y los modelos de gestión vigentes en los espacios de trabajo. Es decir, que la situación de salud de un grupo poblacional específico está condicionada entre otros aspectos por los tipos y características del sistema y servicios de salud 30 como, por ejemplo, en el nivel de resolutividad de las consultas y el acceso. Estas características están muy relacionadas con los modelos de cuidado y los modelos de gestión, que a su vez condicionan la producción, el tipo y la disponibilidad de información en salud.

Paim 31 (p. 567) considera que los modelos asistenciales o modelos de "atención" representan una racionalidad, una lógica, una forma de disponer de los medios de trabajo, que consisten en saberes e instrumentos; y los define como: “... combinações tecnológicas utilizadas pela organização dos serviços de saúde em determinados espaços-populações, incluindo ações sobre o ambiente, grupos populacionais, equipamentos comunitários e usuários de diferentes unidades prestadoras de serviços de saúde com distinta complexidade (postos, centros de saúde, hospitais etc.)".

¿Cómo se interviene en los modelos de cuidado y de gestión? Una forma es jerarquizar el espacio de la gestión local como lugar "del hacer" de los trabajadores de salud 26. Esta línea de trabajo debe jerarquizar también la utilización de tecnologías blandas en el "trabajo vivo en acto" 32,33,34, a fin de desarrollar SIS útiles a la gestión local y comprometidos con la disminución de desigualdades. Esto implica una lógica procesual dónde el trabajo en tanto "hacer", y el conocimiento en tanto "tecnología", son productores de nuevos sujetos (actores) que crean reglas (organización). Así entendido el proceso DICCA es una forma de intervención dirigida al desarrollo y perfeccionamiento de nuevos modelos de cuidado a la salud y de gestión local en salud. Varios elementos apuntan en esa dirección como la integración a los procesos de trabajo, la incorporación de la evaluación y la reflexión, la ampliación del acceso a las informaciones en salud, la comunicación como vía para la participación de profesionales y la población, entre otros.

Finalmente, trabajo y acción nos llevan a Arendt 35 . Esta autora considera a las actividades 
(trabajo y labor) guiadas por los imperativos de la acción instrumental, mientras que el concepto de acción es identificado con la libertad ${ }^{35}$. Es decir, la constitución de un sujeto social consciente de su proceso de trabajo y capaz de recuperar su sentido.

A partir de la visión conjunta de todos los elementos explicitados en esta propuesta es que entendemos debiera contribuir el proceso DICCA a que los SIS den soporte a las decisiones de la gestión.

\section{A modo de cierre y para seguir pensando}

Los problemas señalados no son exclusivos del sector salud, ni de Argentina en particular, y se repiten dentro de diferentes sectores del Estado y la sociedad, así como en otros países de América Latina. Nuestro enfoque está ubicado en la tendencia desarrollada en América Latina denominada Salud Colectiva que levanta una serie de críticas a la Salud Pública tradicional, desarrollada en los países centrales, y contextualiza el proceso salud enfermedad cuidado en la realidad latinoamericana. La Salud Colectiva es considerada un "campo de prácticas" y asume como compromiso la producción de conocimiento para la acción orientada a la eliminación de desigualdades en salud $36,37,38,39$.

Pasados más de 20 años del Seminario sobre Usos y Perspectivas de la Epidemiología organizado por la OPS en Buenos Aires cuyo propósito fue "revisar la práctica epidemiológica actual en el control de las enfermedades, la evaluación de la salud y la planificación de los servicios, y analizar sus implicaciones para el progreso de la investigación, la capacitación en epidemiología y el desarrollo de los servicios" 40 (p. 221) poco se ha avanzado en los SIS como soporte de la gestión.

Pensamos que abordar a los SIS con la lógica DICCA implica también una nueva perspectiva para la epidemiología. Es decir, una epidemiología orientada a la gestión, donde además se rescata la tradición histórica epidemiológica en América Latina ligada a un compromiso de denuncia de desigualdades en salud y orientada a políticas que las reduzcan 39 .

En el desarrollo del trabajo a medida que se avanza en la lógica DICCA se observa la complejidad creciente de los términos. Algunos de ellos presentan una importante polisemia, que abarca un conjunto de definiciones imprecisas y contradictorias entre sí. Muy probablemente esto sea debido a que son términos aplicados a varios campos disciplinares, algunos de ellos con un gran desarrollo en años recientes.

No se trata de indicar una nueva receta que haga "funcionar" a los SIS, sino de alertar sobre la incorporación de elementos como la comunicación y la acción que son imprescindibles, dado que los SIS existen en organizaciones y éstas no deben entenderse solamente desde una lógica racional, ni normativa. Entendemos a los SIS como sistemas abiertos, complejos, y de esta forma la propuesta DICCA se entiende como procesual, donde todos los elementos explicitados son relevantes y se presentan de forma simultánea o superpuesta en la realidad. Es decir, de forma semejante a la concepción de "momentos" (explicativo, normativo, estratégico y táctico operacional) de Matus 20. La integración de DICCA en el proceso de trabajo es la forma de construir el camino, que según Moraes 1, nos lleve de la práctica fragmentada al ejercicio de la ciudadanía

Entendemos que el proceso DICCA debe incorporar la evaluación y la reflexión. Toda acción, o conjunto de ellas, requiere una evaluación. A su vez la evaluación debiera dar soporte a una reflexión sobre esta acción. Esta reflexión debe ser de segundo orden, es decir, en la cuál el sujeto no está separado del objeto. Entendemos que el proceso DICCA descrito anteriormente debe tener este componente reflexivo que permitiría a los trabajadores de salud apropiarse de su proceso de trabajo mediante una concepción creativa, transformadora de la realidad y no alienante del trabajador. En este sentido nos remitimos al concepto de elucidación de Castoriadis 41 que propone pensar lo que se hace y saber lo que se piensa.

La construcción, y reconstrucción, de un SIS es un proceso dialéctico ("supresión, conservación, superación”) en la lógica procesual DICCA. No se debe perder de vista su epigénesis, es decir, que cada estadio nuevo se construye a partir de estadios anteriores. El riesgo de considerarlo como algo dado y sin historia nos llevaría a la "recaída en la inmediatez" 15.

Una de las desigualdades presentes en nuestra sociedad es la relativa al acceso a la información y al conocimiento. La lucha contra ellas es parte de la construcción de ciudadanía social con el fin de construir una sociedad de inclusión. 


\section{Resumen}

La forma tradicional de concepción de los Sistemas de Información en Salud (SIS) presenta innúmeros problemas en dar soporte a la gestión local orientada a la reducción de desigualdades en salud. ¿Cómo diseñar un SIS en el nivel local y cuáles serían sus características? Entendemos a los SIS como sistemas abiertos, complejos y en los que somos parte constitutiva de los mismos. La hipótesis es que un SIS que dé soporte a la gestión local debe ser concebido como un conjunto procesual de elementos formado por dato, información, conocimiento, comunicación y acción (DICCA). El dato es una estructura compleja constituida por cinco componentes. La información es un conjunto de datos procesados; mientras que en la producción de conocimiento existe una comprensión del fenómeno y de apropiación por parte del sujeto. La comunicación es el eje articulador de los conceptos anteriores con la acción. Las acciones estratégicas y comunicativas deben ser jerarquizadas en la gestión local. La propuesta está orientada a que los SIS den soporte a la gestión en la eliminación de desigualdades en salud y en la construcción de una sociedad de inclusión.

Desigualdades en la Salud; Sistemas de Información; Gerencia

\section{Referencias}

1. Moraes IHSD. Informações em saúde: da prática framentada ao exercício da cidadania. São Paulo: Editora Hucitec/Rio de Janeiro: ABRASCO; 1994.

2. Bertalanffy LV. Teoría general de los sistemas: fundamentos, desarrollo, aplicaciones. 10a Reimpresión. México DF: Fondo de Cultura Económica; 1995.

3. Di Tella TS, Chumbita H, Gajardo P, Gamba S. Diccionario de ciencias sociales y políticas. Buenos Aires: Emecé Editores; 2001.

4. Behncke RC. Al pie del árbol [Prefacio]. In: Maturana HR, Varela FG, organizador. El árbol del conocimiento: las bases biológicas del entendimiento humano. 13a Ed. Santiago de Chile: Editorial Universitaria; 1996. p. vi-xxviii.

5. Ibáñez J. El regreso del sujeto: la investigación social de segundo orden. Madrid: Siglo XXI de España Editores; 1994.

6. Mota E, Carvalho DMT. Sistemas de informação em saúde. In: Rouquayrol MZ, Almeida Filho ND, organizadores. Epidemiologia \& saúde. 6a Ed. Rio de Janeiro: Editora MEDSI; 2003. p. 605-28.

7. Castiel LD, Vasconcellos-Silva PR. Precariedades del exceso: información y comunicación en Salud Colectiva. Buenos Aires: Lugar Editorial; 2005.

\section{Colaboradores}

Todos los autores participaron de la idea central del artículo. M. Alazraqui escribió la primera versión, la cual recibió contribuciones originales de los otros autores, E. Mota y H. Spinelli. Todos los autores revisaron y aprobaron la versión final.
8. Rosanvallon P. La nueva cuestión social: repensar el estado providencia. Buenos Aires: Ediciones Manantial; 1995.

9. Galtung J. Teoría y métodos de la investigación social. 5ạ Ed. Buenos Aires: EUDEBA; 1978.

10. Travassos C, Santos SMD, Szwarcwald CL, Barcellos C, Romero D, Bastos FI, et al. Variáveis sociais nos bancos de dados de interesse para a área da saúde. In: Seminário Nacional de Informação e Saúde: o Setor de Saúde no Contexto da Sociedade da Informação. Rio de Janeiro: Fundação Oswaldo Cruz; 2000. p. 35-43.

11. Branco MAF. Sistemas de informação em saúde no nível local. Cad Saúde Pública 1996; 12:267-70.

12. Viacava F. Informações em saúde: a importancia dos inquéritos populacionais. Ciênc Saúde Coletiva 2002; 7:607-21.

13. Mota E. Informações em saúde para a gestão do SUS. Ciênc Saúde Coletiva 2002; 7:637-9.

14. Samaja J. Epistemología y metodología: elementos para una teoría de la investigación científica. 3a Reimpresión. Buenos Aires: EUDEBA; 1997.

15. Samaja J. Epistemología de la salud: reproducción social, subjetividad y transdisciplina. Buenos Aires: Lugar Editorial; 2004. 
16. Castellanos PL. Epidemologia, saúde pública, situação de saúde e condições de vida. Considerações conceituais. In: Barata RB, organizador. Condições de vida e situação de saúde. Rio de Janeiro: ABRASCO; 1997. p. 31-75.

17. Real Academia Española. Diccionario de la lengua española. 22a Ed. http://www.rae.es/ (accedido el Oct/2005)

18. Moraes IHSD. Política, tecnologia e informação em saúde: a utopia da emancipação. Salvador: Casa da Qualidade Editora; 2002.

19. Testa M. Pensar en salud. Buenos Aires: Lugar Editorial; 1993.

20. Matus C. Política, planificación y gobierno. Caracas: Fundación Altadir; 1992.

21. Maturana HR, Varela FG. El árbol del conocimiento: las bases biológicas del entendimiento humano. 13a Ed. Santiago de Chile: Editorial Universitaria; 1996.

22. Searle JR. Actos de habla: ensayo de filosofía del lenguaje. 3a Ed. Madrid: Ediciones Cátedra; 1990.

23. Flores F. Creando organizaciones para el futuro. 5a Ed. Santiago de Chile: Dolmen Ediciones; 1997.

24. Flores F. Inventado la empresa del siglo XXI. 10a Ed. Santiago de Chile: Dolmen Ediciones; 1997.

25. Habermas J. Teoría de la acción comunicativa: I. Racionalidad de la acción y racionalización social. Madrid: Taurus Humanidades; 1999.

26. Spinelli H, Urquía M, Bargalló ML, Alazraqui M. Equidad en salud: teoría y praxis. In: Spinelli $\mathrm{H}$, organizador. Salud colectiva: cultura, instituciones y subjetividad. Epidemiología, gestión y políticas. Buenos Aires: Lugar Editorial; 2004. p. 247-77.

27. Matus C. Chipancé, Machiavello y Gandhi: estrategias políticas. Caracas: Fundación Altadir; 1995.

28. Testa M. Saber en salud: la construcción del conocimiento. Buenos Aires: Lugar Editorial; 1997.

29. Paim JS. Por um planejamento das práticas de saúde. Ciênc Saúde Coletiva 1999; 4:243-8.

30. Tigre CH, Plaut R, Libel M, Castellanos PL. La práctica epidemiológica en los sistemas de servicios de salud. Educ Méd Salud 1990; 24:306-20.
31. Paim JS. Modelos de atenção e vigilancia da saúde. In: Rouquayrol MZ, Almeida-Filho ND, organizadores. EpidemiologIa \& saúde. 6a Ed. Rio de Janeiro: Editora MEDSI; 2003. p. 567-86.

32. Merhy EE. Em busca do tempo perdido: a micropolítica do trabalho vivo em saúde. In: Merhy EE, Onocko R, organizadores. Agir em saúde: um desafio para o público. São Paulo: Editora Hucitec/ Buenos Aires: Lugar Editorial; 1997. p. 71-112.

33. Merhy EE, Chakkour M, Stéfano E, Stéfano ME, Santos CM, Rodrigues RA, et al. Em busca de ferramentas analisadoras das tecnologias em saúde: a informação e o dia a dia de um serviço, interrogando e gerindo trabalho em saúde. In: Merhy EE, Onocko R, organizadores. Agir em saúde: um desafio para o público. São Paulo: Editora Hucitec/ Buenos Aires: Lugar Editorial; 1997. p. 113-50.

34. Merhy EE. Saúde: a cartografia do trabalho vivo. São Paulo: Editora Hucitec; 2002.

35. Arendt H. A condição humana. 7ạ Ed. Rio de Janeiro: Forense Universitária; 1995.

36. Paim JS, Almeida Filho ND. Saúde coletiva: uma "nova saúde pública" ou campo aberto a novos paradigmas? Rev Saúde Pública 1998; 32:299-316.

37. Paim JS, Almeida-Filho ND. A crise de saúde pública e a utopia da saúde coletiva. Salvador: Casa da Qualidade Editora; 2000.

38. Barreto ML, Almeida Filho N, Breilh J. Epidemiology is more than discourse: critical thoughts from Latin America. J Epidemiol Community Health $2001 ; 55: 158-9$

39. Barreto ML. The globalization of epidemiology: critical thoughts from Latin America. Int J Epidemiol 2004; 33:1132-7.

40. Organización Panamericana de la Salud. Usos y perspectivas de la epidemiología. In: Seminario sobre Usos y Perspectivas de la Epidemiología. Washington DC: Organización Panamericana de la Salud, Organización Mundial de la Salud; 1984.

41. Castoriadis C. Figuras de lo pensable. Buenos Aires: Fondo de Cultura Económica; 2001.

Recibido el 25/Nov/2005

Versión final presentada el 11/Abr/2006

Aprobado el 02/May/2006 